\title{
Activity of etravirine on different HIV-I subtypes: week 48 data of the pooled DUET trials and in vitro susceptibility in treatment-naïve patients
}

\author{
J Vingerhoets*1, H Azijn ${ }^{1}$, L Tambuyzer ${ }^{1}$, I Dierynck ${ }^{1}$, S De Meyer ${ }^{1}$, \\ L Rimsky $^{1}$, M Peeters ${ }^{1}$, G De Smedt ${ }^{1}$, MP de Béthune ${ }^{1}$ and G Picchio ${ }^{2}$
}

Address: ${ }^{1}$ Tibotec BVBA, Mechelen, Belgium and ${ }^{2}$ Tibotec Inc, Yardley, USA

* Corresponding author

from Ninth International Congress on Drug Therapy in HIV Infection

Glasgow, UK. 9-13 November 2008

Published: 10 November 2008

Journal of the International AIDS Society 2008, I I (SuppI I):PI89 doi:I0.I I86/I758-2652-II-SI-PI89

This abstract is available from: http://Www.jiasociety.org/content/I I/SI/PI89

(C) 2008 Vingerhoets et al; licensee BioMed Central Ltd.

\section{Purpose of the study}

Etravirine (ETR, TMC125) has shown good in vitro activity against primary HIV-1 group M isolates from different subtypes and has demonstrated durable efficacy in treatment-experienced, HIV-1 infected patients in the Phase III DUET trials. In vivo efficacy and in vitro activity of ETR against different HIV-1 subtypes were further investigated.

\section{Methods}

DUET patients were randomized 1:1 to ETR (200 mg BID) or placebo, both with a background regimen of NRTIs, darunavir/ritonavir and optional enfuvirtide. Subgroup analyses of the effect of HIV-1 subtype on the proportion of patients with viral load (VL) <50 HIV-1 RNA copies/mL (TLOVR imputation algorithm) were conducted on pooled week 48 data. Genotype/subtype and phenotype determinations were performed using the Virco ${ }^{\circledR}$ TYPE HIV-1 and Antivirogram ${ }^{\mathrm{TM}}$ assays, respectively. The effect of HIV-1 subtype on ETR fold change in EC50 value (FC) was analyzed in HIV-1 recombinant clinical isolates from treatment-naïve patients enrolled in other Tibotec trials ( $\mathrm{n}$ $=872)$ that included $49 \%$ of HIV-1 subtype non-B $(18 \%$ CRF01_AE; $16 \% \quad$ C; $5 \%$ A1; 3\% CRF12_BF; $2 \%$ CRF02_AG; $1 \% \mathrm{~F} 1 ; 3 \%$ other).

\section{Summary of results}

In DUET, HIV-1 subtype was available for 594 and 595 patients in the ETR and placebo arms, respectively. The majority of these $(93.8 \%)$ harboured HIV-1 subtype B.
Among the non-B subtypes, CRF12_BF (2.1\%), F1 $(1.2 \%)$, and CRF02_AG $(0.8 \%)$ were most prevalent. Baseline disease characteristics (VL, CD4, ETR FC, DRV FC, PSS) were similar between patients with different subtypes, except for a higher number of sensitive NRTIs used in those with HIV-1 subtype non-B. In the ETR arm, virological responses at week 48 were $59.9 \%$ (336/561) for HIV-1 subtype B vs. $72.7 \%$ (24/33) for all other HIV-1 subtype non- $\mathrm{B}$, as compared to an overall response of $60.6 \%$.

These data were further supported by in vitro results that indicated a comparable median (IQR) ETR FC in virus isolates from treatment-naïve patients infected with subtype B or non-B (1.1, $0.8-1.6$ or $1.2,0.81 .7)$, respectively.

\section{Conclusion}

In the DUET studies, ETR was equally effective in suppressing viral replication in patients infected with HIV-1 subtype B or non-B. Furthermore, both subtype B and non-B HIV-1 recombinant clinical isolates from treatment-naïve patients exhibited comparable levels of in vitro phenotypic susceptibility to ETR. These results confirmed the broad activity of ETR against HIV-1. 